\title{
Cystic fibrosis screening using the College panel: Platform comparison and lessons learned from the first 20,000 samples
}

Charles M. Strom, MD, PhD, Donghui Huang, PhD, Arlene Buller, PhD, Joy Redman, MS, Beryl Crossley, MD, Ben Anderson, Tom Entwistle, and Weimin Sun, MD, PhD

\begin{abstract}
Purpose: To determine the accuracy of two commercially available kits for cystic fibrosis (CF) genotyping and determine allele frequencies for the ACMG/ACOG recommended mutations. Methods: A total of 1,040 consecutive analyses using Roche CF Gold Strips and the ABI CF Genotyper were performed. Subsequently we performed analyses of 20,103 samples. Results: Both kits accurately determined CF genotypes. The I148T mutation was found $>100$ times more frequently in carrier screening than in CF patients. Asymptomatic patients were identified who are compound heterozygotes for delta F508 and I148T. Four of 13 patients heterozygous for delta F508 and the IVS8-5T polymorphism had some symptoms of CF. Conclusion: Accurate and timely analysis can be performed for the ACMG CF panel. I148T is a low penetrance CF allele. Genet Med 2002:4(4):289-296.
\end{abstract}

Key Words: cystic fibrosis, carrier screening, population screening, allele frequencies

Cystic fibrosis (CF) is one of the most common recessive genetic diseases in North America. In the March/April edition of this Journal, the Subcommittee on Cystic Fibrosis Screening of the American College of Medical Genetics (ACMG) published laboratory standards for population-based CF carrier screening. ${ }^{1}$ The Committee recommended a core screening panel of 25 mutations with reflex testing under certain circumstances to four polymorphisms. These recommendations were based on the published frequencies of mutations observed in CF patients and other more subtle considerations such as a reluctance to screen for mutations causing congenital bilateral absence of the vas deferens (CBAVD). In October 2001, the American College of Obstetrics and Gynecology (ACOG) published its anticipated recommendation to College members to offer CF carrier detection to pregnant women. ${ }^{2}$

In preparation for the release of the ACOG clinical practice guideline, we evaluated two of the commercially offered analytic platforms available as Analyte Specific Reagents (ASR) by performing blinded side by side analysis of 1,040 samples and determined that both performed acceptably.

Our laboratory began screening for the College recommended panel in July 2001. To date, more than 20,000 samples have been analyzed. Many interesting observations are reported here with respect to test volumes, mutation frequencies, and accuracy of commercially available products. We also dis-

\footnotetext{
From the Molecular Genetics Laboratory, Quest Diagnostics Nichols Institute, San Juan Capistrano, California.

Charles M. Strom, MD, PhD, Molecular Genetics Laboratory, Quest Diagnostics Nichols Institute, 33608 Ortega Highway, San Juan Capistrano, CA 92690.

Received: February 25, 2002.

Accepted: April 25, 2002.
}

DOI: 10.1097/01.GIM.0000021414.62685.9F cuss clinical problems that have arisen. Our data and discussion provide insight into potential problems and solutions as CF population screening becomes integrated into standard obstetric care.

\section{MATERIALS AND METHODS}

\section{Patient population}

In the period between July 1, 2001, and December 1, 2001, 20,103 consecutive samples submitted for CF DNA testing were analyzed for the ACMG recommended panel of mutations. Although information such as patient ethnicity and pertinent family history are requested for each patient, in practice, this information was only sporadically provided. When appropriate, physicians were contacted to obtain clinical information regarding patients with interesting genotypes. Because the indication was not routinely provided, we have no way to determine the number of tests performed for carrier detection versus mutation detection in a patient with $\mathrm{CF}$, or infertility evaluations. Therefore, all the data were analyzed together, assuming that the overwhelming majority of the 20,000 tests were performed for carrier detection.

\section{DNA purification}

DNA was extracted on Qiagen Robots using conditions specified by the manufacturer. If assays required manual extraction of DNA, phenol chloroform extraction and ethanol precipitation were performed by standard techniques.

\section{CF mutation assays}

Two assays for CF mutations were performed. The Linear Array CF Gold 1.0 Panel (LAp) ASR kits were purchased from Roche Molecular Biochemicals. These reagents consist of a 15- 
plex polymerase chain reaction (PCR) master mix containing biotinylated primers. PCR was performed in a MJR Model PCT 225 thermocycler under conditions recommended by the manufacturer. Hybridization of sample PCR product to the CF strips was followed by conjugation using the ASR reagents provided by the manufacturer. After washing the strips with proprietary reagents using proprietary conditions, colorimetric detection of conjugated hybridized PCR products was performed using manufacturer supplied reagents and conditions. Hybridizations, detection, and washing steps were carried out manually in batches of 48 . Scoring was performed visually and reviewed by a laboratory supervisor and laboratory director before reporting. The presence of the IVS8-5T/7T/9T and I506 V, I507 V, F508C polymorphisms were detected and reported for the initial 20,103 patients before our introduction of a polymorphic reflex test.

The second ASR was supplied by Applied Biosystems (ABI CF Genotyper V 2.0). This reagent consists of a multiplex PCR master mix followed by an oligonucleotide ligation assay (OLA) with detection performed on an ABI 3100 capillary sequencer. The ABI kit does not detect three mutations in the ACMG panel: $3120+1 \mathrm{G} \rightarrow \mathrm{A}, 2184 \mathrm{delA}$, and I148T; it detects nine mutations not in the ACMG panel: 2183delA, $3849+$ $4 \mathrm{~A} \rightarrow \mathrm{G}, 3905 \mathrm{ins} \mathrm{T}, \mathrm{Q} 493 \mathrm{X}, \mathrm{S} 549 \mathrm{~N}, \mathrm{~S} 549 \mathrm{R}, \mathrm{V} 520 \mathrm{~F}, \mathrm{Y} 122 \mathrm{X}$, and $\mathrm{R} 347 \mathrm{H}$. Therefore, we designed a supplemental assay containing the three ACMG panel mutations and an additional mutation, D1270N, that we had detected with an unexpectedly high frequency using our previous home brew assay. The supplemental assay consists of a multiplex PCR followed by a single nucleotide primer extension reaction (SNP-IT) licensed from Orchid Biosystems using an ABI SnapShot kit. The PCR primers and extension primers are listed in Table 1. The PCR cycling protocol was as follows: 95 deg 15 minutes, followed by 31 cycles of denaturing at $94 \mathrm{deg}$ for 10 seconds (ramping of 0.5 deg/second), annealing at 56 deg for 10 seconds (ramping 0.3 $\mathrm{deg} /$ second), and polymerization at $72 \mathrm{deg}$ for 15 seconds (ramping $0.5 \mathrm{deg} / \mathrm{second}$ ). Cycling was followed by a 5 -minute chain elongation step at $72 \mathrm{deg}$ and cooling to $4 \mathrm{deg}$. The SnapShot reaction was carried out as recommended by the manufacturer. The products of the primer extension reaction were analyzed by capillary electrophoresis on an ABI 3100 sequencer, and allele assignments were made using Genotyper (Tm ABI) software. The population used for the platform comparison consisted of 1,040 consecutive samples submitted for CF DNA testing to our reference laboratory.

\section{RESULTS}

\section{Test volumes}

Figure 1 is a graphical representation of the weekly CF test volumes in our laboratory. After the October 2001 publication of the ACOG recommendations, there was a sharp increase in the CF genotyping test volume. Our average weekly test volumes were 834 for the weeks beginning July 2 to September 24, 2001. A steep rise began in early October and has continued to a volume of 3,418 for the week beginning February 11, 2002. This quadrupling of test volumes confirms the anticipated impact of new practice guidelines on test volumes and reinforces the importance of giving clinical testing laboratories advance notification of such announcements so that preparations can be made to handle the increase in test volumes.

\section{Assay validation and performance}

Before introducing the test into operation, a validation was performed using a test panel consisting of 94 samples of known genotype and 2 negative controls. The genotypes included normal controls and at least one heterozygote for each of the 25 mutations and 6 polymorphisms in addition to compound

Table 1

PCR primers and sequencing primers for the supplemental CF assay

\begin{tabular}{ll}
\hline Amplicon & \multicolumn{1}{c}{ PCR primer sequence $\left(5^{\prime} \rightarrow 3^{\prime}\right)$} \\
\hline Exon $4-\mathrm{F}$ & GCGGTCCCAAAAGGGTCAGTTGTAGGAAGTCACCAAAG \\
Exon $4-\mathrm{R}$ & GCGGTCCCAAAAGGGTCAGTCGATACAGAATATATGTGCC \\
Exon $13-\mathrm{F}$ & GCGGTCCCAAAAGGGTCAGTAGGTAGCAGCTATTTTTATGG \\
Exon $13-\mathrm{R}$ & GCGGTCCCAAAAGGGTCAGTTAAGGGAGTCTTTTGCACAA \\
Intron $16-\mathrm{F}$ & GCGGTCCCAAAAGGGTCAGTGCAATTTTGGATGACCTTC \\
Intron $16-\mathrm{R}$ & GCGGTCCCAAAAGGGTCAGTGAGGGTTGAAGTCCTGTCTA \\
Exon $20-\mathrm{R}$ & GCGGTCCCAAAAGGGTCAGTTCCTTTTGCTCACCTGTGGT \\
Exon $20-\mathrm{F}$ & GCGGTCCCAAAAGGGTCAGTCTGGATCAGGGAAGA \\
Mutation & \\
I148T & Sequencing primer $\left(5^{\prime} \rightarrow 3^{\prime}\right)$ \\
$2184 \mathrm{delA}$ & GATCGATCTGTCTCCTGGACAGAAACAAAAAA \\
D1270N & GACTGATCGATCGTTATTGAATCCCAAGACACACCAT \\
$3120+1$ G $>$ A & GACTGATCGATCGATCCCTCTTACCATATTTGACTTCATCCAG \\
\hline
\end{tabular}




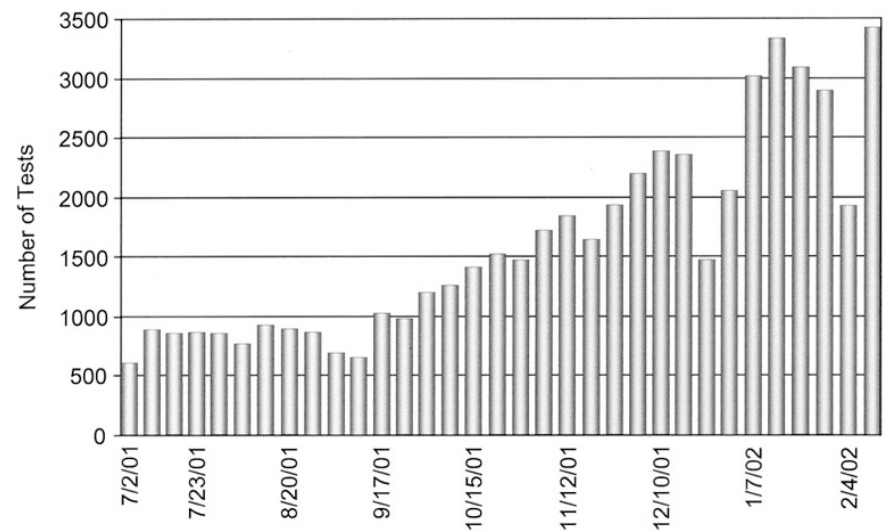

Fig. 1 Weekly test volumes submitted to Quest Diagnostics for CF DNA analysis. X axis, date; $\mathrm{Y}$ axis, number of tests.

heterozygotes and homozygotes for delta F508. The panel was aliquotted into three 96-well PCR plates, with each plate having a different arrangement of the control samples. The laboratory technologists and directors were blinded to the locations of each sample in each plate. One technologist performed the assay on two of the plates and a different technologist performed the assay on the third plate. The samples were scored blindly and then compared with the expected genotypes. All samples were correctly genotyped.

Once in operation, we monitor monthly the number of samples that need to be repeated. For blood samples the repeat rate varies from approximately $1 \%$ to $3 \%$. In the series of more than 20,000 patients a failure rate of 8 per 10,000 was observed. This of course applies only to samples that reached the laboratory intact. For direct amniocentesis samples, approximately $10 \%$ of samples cannot be analyzed, but genotypes were eventually successful for all samples using tissue culture material. There were an insufficient number of chorionic villi samples to reach any conclusion regarding repeat rates.

\section{Platform comparison}

To our knowledge, the Roche CF Gold LAp strip assay (Roche Molecular Systems) is the only commercial platform available as an ASR to be fully compliant with the ACMG mutation panel. This is a fluid area and several new products are expected to be introduced in the near future. As part of our validation program, we believed it was important to ascertain the accuracy of these strips for genotype assignment. We supplemented another commercially available ASR, the ABI CF Genotyper Version 2.0 with a proprietary supplemental assay for our comparison study (see "Materials and Methods"). Any discrepancies were resolved by sequencing the relevant PCR product. Of note is that two companies are planning the release of ACMG compliant assays in the near future, Innogenetics and the ABI CF Genotyper Version 3.0.

A total of 1,040 consecutive samples were assayed by both the Roche LAp assay and the combination OLA/supplemental assay. All samples were successfully analyzed using both platforms. Of the 1,040 samples, 20 (1.9\%) of the Roche series and
27 (2.6\%) of the ABI series required a repeat analysis to obtain interpretable results. This difference was not statistically significant (chi square $=0.78 ; P=0.38$ ), and both levels were within our acceptable laboratory standards of repeat rates $<5 \%$.

There was only one true genotyping discrepancy observed in this series. The LAp assay identified one sample as a compound heterozygote for delta F508 and the $1898+1 \mathrm{G} \rightarrow$ A mutation. The ABI assay identified this patient to be heterozygous delta F508/wild-type. Sequence analysis revealed the patient to be heterozygous for delta F508 and $1898+1 \mathrm{G} \rightarrow \mathrm{C}$ mutation. Both the $\mathrm{G}$ to $\mathrm{A}$ and the $\mathrm{G}$ to $\mathrm{C}$ transversion have been described as CF mutations. ${ }^{3}$ Upon review of the LAp strips for this patient, the intensity of the $1898+1 \mathrm{G} \rightarrow \mathrm{A}$ band was noted to be lighter than the other mutant bands for other alleles in the same batch of analyses but was clearly positive. Because neither the Roche assay nor the ABI assay are designed to detect the $1898+1 \mathrm{G} \rightarrow \mathrm{C}$ mutation, it is difficult to fault either assay in this instance.

The only other differences between the assays were in mutation and polymorphic panels analyzed by the respective assays. Four patients were found to be heterozygous for the D1270N by our supplemental assay. No patients with the nine additional mutations detected by the OLA kit were identified. The ABI/supplemental assay identified a patient as a compound heterozygote for I148T and D1270N, whereas the Roche assay does not detect D1270N.

We chose the LAp assay for future testing, although the OLA assay enjoys two significant advantages. We were able to automate the OLA assay, and as a result, labor costs are significantly reduced. In addition, the ABI 3100 capillary sequencer has automated allele calling software, which saves interpretation time and eliminates the necessity to manually enter results. These advantages were negated by the fact that the current OLA kit requires a supplemental test to be compliant with the ACMG recommendations and the OLA kit does not contain the reflex polymorphisms so that some patient samples would require three separate tests to generate a report.

\section{Assay failure rate}

The LAp assay performed well on both prenatal samples and blood samples. If no result was obtained on the initial analysis, DNA extraction was repeated and the analysis repeated. If this also failed, a manual DNA extraction was performed and a third analysis attempted. If this third analysis failed, a "no result" was reported. Table 2 demonstrates that only 17 patients (34 chromosomes) for a rate of 8 samples per 10,000 could not be genotyped.

Short turnaround times are of vital importance for prenatal patients. For $90 \%$ of cases we were able to provide results $<4$ days after receipt of blood samples.

\section{Mutation frequencies}

Table 2 shows a summary of mutation frequencies observed in the series of 20,103 consecutive patients analyzed with the LAp assay. In this series there were only 51 samples that contained more than $1 \mathrm{CF}$ mutation. Clinical data for some of 
Table 2

Summary of CF mutations in 20,103 consecutive patients

\begin{tabular}{|c|c|c|c|c|c|}
\hline Mutation & $\begin{array}{c}\text { Carrier } \\
\text { detection: No. } \\
\text { of CF } \\
\text { chromosomes }^{\text {detected }}{ }^{a}\end{array}$ & $\begin{array}{c}\text { Carrier } \\
\text { detection: \% } \\
\text { of CF } \\
\text { chromosomes }\end{array}$ & $\begin{array}{l}\text { CF patients }{ }^{b} \text { : } \\
\text { No. of } \\
\text { chromosomes } \\
\text { from } 2,920 \\
\text { patients }\end{array}$ & $\begin{array}{c}\text { CF patients }{ }^{b} \text { : } \\
\% \text { of CF } \\
\text { chromosomes }\end{array}$ & $\begin{array}{l}\text { Excess } \\
\text { carrier/ } \\
\text { patient }\end{array}$ \\
\hline delta F508 & 595 & 63 & 3,539 & 60 & 1.0 \\
\hline $\mathrm{R} 117 \mathrm{H}$ & 75 & 9.1 & 35 & 0.60 & 15.1 \\
\hline I148T & 64 & 7.7 & 4 & 0.068 & 113 \\
\hline W1282X & 58 & 7.0 & 76 & 1.3 & 5.4 \\
\hline G542X & 34 & 4.1 & 102 & 1.7 & 1.8 \\
\hline N1303K & 19 & 2.0 & 78 & 1.3 & 1.5 \\
\hline $3849+10 \mathrm{~KB}$ & 18 & 2.2 & 25 & 0.42 & 5.2 \\
\hline \multicolumn{6}{|l|}{$\mathrm{C} \rightarrow \mathrm{T}$} \\
\hline G551D & 17 & 2.1 & 134 & 2.9 & 0.72 \\
\hline R533X & 10 & 1.2 & 69 & 1.2 & 1 \\
\hline $\mathrm{R} 560 \mathrm{~T}$ & 8 & 0.97 & 21 & 0.36 & 2.8 \\
\hline $2789+5 \mathrm{G} \rightarrow \mathrm{A}$ & 7 & 0.85 & 44 & 0.75 & 1.1 \\
\hline $621+1 \mathrm{G} \rightarrow \mathrm{T}$ & 7 & 0.85 & 52 & 0.89 & 0.95 \\
\hline R1162X & 5 & 0.60 & 23 & 0.39 & 1.5 \\
\hline A455E & 4 & 0.48 & 15 & 0.26 & 1.8 \\
\hline $711+1 \mathrm{G} \rightarrow \mathrm{T}$ & 4 & 0.48 & 7 & 0.12 & 4.0 \\
\hline $1717-1 \mathrm{G} \rightarrow \mathrm{A}$ & 4 & 0.48 & 45 & 0.77 & 0.62 \\
\hline G85E & 3 & 0.36 & 20 & 0.34 & 1.1 \\
\hline $3120+1 \mathrm{G} \rightarrow \mathrm{A}$ & 3 & 0.36 & 39 & 0.67 & 0.54 \\
\hline $1898+1 \mathrm{G} \rightarrow \mathrm{A}$ & 3 & 0.36 & 28 & 0.48 & 0.75 \\
\hline delta I507 & 3 & 0.36 & 35 & 0.60 & 0.60 \\
\hline $3659 \mathrm{del} \mathrm{C}$ & 2 & 0.24 & 25 & 0.42 & 0.57 \\
\hline $2184 \mathrm{del} A$ & 2 & 0.24 & 17 & 0.29 & 0.82 \\
\hline R347P & 1 & 0.12 & 20 & 0.34 & 0.35 \\
\hline R334W & 1 & 0.12 & 20 & 0.34 & 0.35 \\
\hline 1078 DEL T & 0 & & 1 & 0.017 & \\
\hline
\end{tabular}

${ }^{a}$ Some chromosomes were from homozygotes.

${ }^{b}$ From Heim et al. ${ }^{4}$

these patients was obtained and many were asymptomatic and testing was performed for obstetrical carrier detection. Therefore, we did not exclude these data from the analyses. Table 3 is

Table 3

Prevalence of polymorphisms in 20,103 patients

\begin{tabular}{lrc}
\hline Polymorphism & No. & \% of all chromosomes \\
\hline IVS8-5T & 1,846 & 4.6 \\
F508C & 67 & 0.17 \\
I506V & 29 & 0.072 \\
I507V & 1 & 0.00025 \\
\hline
\end{tabular}

a summary of the polymorphism frequencies in the same population.

For comparison, Table 2 also includes published data from Heim et al. ${ }^{4}$ of mutation frequencies observed in 2,920 CF patients $(5,840$ chromosomes) in a similar U.S. pan ethnic population. In general, the frequencies observed in our population of patients tested mainly for carrier status were congruent with the observations made in CF patients with three notable exceptions. The mutation $\mathrm{R} 117 \mathrm{H}$ was present on $9.1 \%$ of $\mathrm{CF}$ chromosomes detected in our series, more than 15 -fold higher than its prevalence in CF patients (Table 2). This observation is not surprising as $\mathrm{R} 117 \mathrm{H}$ is known to be associated with a milder phenotype or CBAVD rather than classical CF 
unless associated with the IVS8-5T allele. Therefore, it is expected that $\mathrm{R} 117 \mathrm{H}$ would be more prevalent in a mostly asymptomatic, primarily female population tested for carrier status than in a population of CF patients.

More striking was the 113-fold increase in the prevalence of I148T in our population when compared with the CF patient population. The I148T mutation accounted for $7.7 \%$ of $\mathrm{CF}$ chromosomes detected in our screened population versus $0.068 \%$ of CF chromosomes in CF patients. ${ }^{4}$ This observation may indicate that this mutation is not a true mutation but simply a polymorphism or an extremely mild CF allele with incomplete penetrance. We observed two asymptomatic adult pregnant women who are compound heterozygotes for delta F508 and I148T. A preliminary report noted five such unaffected compound heterozygous individuals. ${ }^{5}$ Confirmation of these observations may have important implications for genetic counseling of patients and couples found to carry this mutation. We did not obtain any information regarding race or phenotype for the other 62 patients who tested positive for one copy of the I148T mutation and negative for all other tested mutations.

In the platform comparison study, the mutation D1270N was included because of our observation of a relatively high prevalence of this mutation in our screened population when patients were analyzed using a different assay. The D1270N mutation was observed in 5 of 2,080 chromosomes analyzed and accounted for $14 \%$ of all CF chromosomes observed in this series of patients. Comparisons with the published frequency of $0.068 \%{ }^{4}$ reveal that $\mathrm{D} 1270 \mathrm{~N}$ is present 205 times more commonly in our population of carrier screening patients than in CF patients. This finding suggests that this mutation, similar to I148T, is a low penetrance allele.

No other allele was found to be in $>10$-fold excess in the carrier population when compared with the CF patient population. W1282X was the only mutation to even approach this level with a 9.1-fold higher incidence. Further data will be required before conclusions can be reached regarding the penetrance of such alleles. Because the $\mathrm{R} 117 \mathrm{H}$ allele is present at a 15-fold higher frequency in the screened population versus the patient population, this may be an appropriate threshold to begin further investigation regarding penetrance.

The D1270N allele is not part of the ACMG recommended panel allele. It is found primarily in Hispanic populations. In CF patients, three Hispanic chromosomes and only one Caucasian chromosome were found to have this mutation. ${ }^{4}$ In our series, the D1270N allele was analyzed for only 1,040 patients and 2,080 chromosomes because it was included in our supplemental OLA assay and not in the LAp assay. This allele was present in 5 of the $36 \mathrm{CF}$ chromosomes (14\%), yielding a prevalence rate of 159 times that of the CF patient cohort. ${ }^{4}$ In addition, one man was identified who is a compound heterozygote for D1270N and I148T and completely asymptomatic at age 26 . He and his wife had only recently been trying to conceive, so his fertility is not yet known. This finding provides some further evidence that one or both of these mutations may not be true CF mutations.

\section{T or not 5T}

The LAp strips detect all polymorphisms recommended as reflex tests by the ACMG, including the IVS8-5T/7T/9T polymorphism in intron 8 . Aberrant splicing of mRNA caused by the presence IVS8-5T has been associated with both pulmonary symptoms and CBAVD.6,7 Several studies have demonstrated that the IVS8-5T allele in combination with other CF alleles, including $\mathrm{R} 117 \mathrm{H}$, has been associated with CBAVD. ${ }^{8-10}$ The ACMG recommends analysis of the IVS8-5T only in the presence of R117H because IVS8-5T in cis with R117H makes $\mathrm{R} 117 \mathrm{H}$ a full CF allele. It is important to note that the ACMG recommendations are for population carrier screening. However, CF DNA testing can be ordered for other reasons in symptomatic patients. Physicians caring for patients with CBAVD are often specifically looking for the IVS- 8 genotype because homozygosity for $5 \mathrm{~T}$ has been associated with CBAVD. Often geneticists are interested in the IVS- 8 genotype for the purposes of linkage analysis in a family. Because a reference laboratory has no way of knowing why a test was ordered, we choose to allow the physician to select which test he or she would like. We encourage obstetricians, family practitioners, sperm bank directors, and midwives to order the reflex test which reports the IVS-8 genotype only in presence of another CF mutation. This is slightly different from the ACMG recommendation of reporting only in the presence in $\mathrm{R} 117 \mathrm{H}$, but we believed there was a sufficient amount of published data regarding mildly affected individuals heterozygous for other CF mutations and $5 \mathrm{~T}$ to warrant this inconsistency. ${ }^{6-10}$ In fact we identified four patients who had mild symptoms of CF who are compound heterozygotes for delta F508 and 5T (see below).

We offer a second test for physicians such as urologists, fertility specialists, and geneticists who wish to determine the IVS-8 genotype in which the presence of the 5T allele is reported in all cases. The laboratory procedures are identical for the two tests. The genotype, including the IVS8-5T, is entered into the computer. If the ordered test was the reflex test, the computer ignores the presence of a IVS8-5T allele in the absence of a CF mutation and prints out a wild-type result. If the nonreflex test is ordered, the computer generates a report that includes the IVS8-5T, if present, under all circumstances. This solution seems to satisfy almost all of our referring physicians.

\section{Compliance issues}

This type of selective reporting is consistent with current compliance practices in clinical laboratories. For example, if a clinician orders a hemoglobin, most laboratories will subject the sample to an automated analyzer that determines not only the hemoglobin, but the hematocrit and leukocyte count. The physician receives only the requested hemoglobin result even though there may be an abnormality in the patient's white blood count. The laboratory incurs no risk of liability and as a matter fact is prohibited from reporting a result that was not ordered by the physician, regardless of the potential clinical relevance of the unreported result. This system has the advan- 
tage of storing the IVS8-5T data in the computer system should the physician or a consulting physician wish to know the IVS8-5T status at a later date. We believe this is a preferred solution to the alternative of cutting the polymorphic LAp locations off the strip. The latter alternative would increase turnaround time in the event a reflex test is needed as another reaction will be required and represents a logistic tangle for the laboratory in trying to sort patients by which test was ordered.

Our solution is fully compliant with all current federal and state regulations, which require laboratories to report and bill only for specific tests ordered by physician but does not preclude laboratories from using instrumentation and/or methods that perform additional tests as long as the unordered tests are neither reported nor billed for.

In our series, 33 patients were found to be heterozygous for delta F508 and the IVS8-5T polymorphism. In all cases, the patients also had a 9T allele. Because the 9T allele is almost always associated in cis to delta F508, it can be assumed that, for almost all these patients, the IVS8-5T is in trans to the delta F508 mutation. We attempted to obtain clinical information for all these patients. To date we were successful for 13 patients. Nine patients (all female) were asymptomatic. Four patients had symptoms consistent with mild CF. A 6-month-old male infant had several surgeries for malrotation and meconium ileus. He had a normal sweat test and no lung symptoms. A female patient had recurrent pneumonias with bronchiectasis and no pancreatic problems. Another female patient had severe asthma, and a male patient had CBAVD. With this small data set it is not possible to determine with certainty whether IVS8-5T in trans is the true cause of these symptoms, because it is possible that another, undetected CF mutation is responsible. However, that $30 \%$ of compound heterozygous patients for delta F508 and IVS8-5T and all of the male patients with this genotype have symptoms attributable to defective cystic fibrosis transmembrane regulator protein (CFTR) suggest that the IVS8-5T allele may be a sex-influenced low-penetrance CF allele. Experimental data has determined that IVS8-5T in cis can lower the amount of functional CFTR transcript. ${ }^{6}$

\section{Other polymorphisms}

The polymorphisms F508C, I506V, and I507V are important because they can prevent the amplification and/or detection of the wild-type sequence in exon 10. If a patient is a compound heterozygote for delta F508 or delta I507, certain analytic methods, including the Lipa strips can falsely appear to be homozygous for the mutations when, in fact, the patients are heterozygous for a normal allele and a CF allele. Misdiagnosis was avoided for two patients because of the analysis of these polymorphisms. A patient who appeared to be homozygous for delta F508 was a compound heterozygote for delta F508 and F508C. A second patient was positive for the F508C polymorphism and had no reaction with delta F508, delta I507 or with the wild-type for those two mutations, indicating that this patient is probably homozygous for F508C and not a carrier of CF. Because there are no other exon 10 alleles analyzed in the College panel, it is not possible to determine with cer- tainly if the observed result was due to PCR failure of one of the exon 10 alleles and the patient is truly heterozygous for F508C. No clinical information was available for this patient. It is important to note that the OLA kit does not suffer from this potential ambiguity so that it is unnecessary to measure these three polymorphisms in that assay. The ACMG recommends that these polymorphisms only be reported in the presence of homozygosity for delta F508 or delta I507.

\section{Prenatal diagnosis}

Sixteen prenatal diagnoses were performed because of the presence of the IVS8-5T polymorphism in one or both parents. In nine cases, one parent carries a CF mutation and the other parent the IVS8-5T, but in four cases, both parents have the IVS8-5T and neither parent has a CF mutation. In one such case, the mother is homozygous for IVS8-5T and, as expected, asymptomatic. In three cases, one parent has the IVS8-5T and the other is negative for CF mutations and the IVS8-5T polymorphism. Although it is possible that some of these patients have another indication for prenatal sampling other than CF, the possibility is raised that the significance of the IVS8-5T polymorphism is misinterpreted by clinicians and might be the cause of unnecessary prenatal diagnostic procedures, heightening the importance of offering a prenatal screening test that does not report the IVS8-5T polymorphism unless another CF mutation is present. Because it is impossible to assure obstetricians that a child born with a CF mutation and IVS8-5T in trans or homozygous IVS8-5T will be normal, many will elect to offer a prenatal diagnosis to couples at risk for such fetuses rather than risk the potential liability of the birth of an affected child. Once the conundrum of IVS8-5T has been raised, it is difficult to completely reassure the physician or couple. Therefore, we believe it is better to perform obstetrical screening using the College recommended reflex guidelines so the issue is not raised at all. This also emphasizes the importance of educating ordering physicians regarding the subtleties of the genetic counseling issues involved in CF carrier screening. Only four $(8 \%)$ of the fetal samples came from couples who both carry CF mutations, in seven cases no indication was provided, and the remaining prenatal samples were sent because of positive family history.

\section{DISCUSSION}

As the result of a cooperative program between ACMG and ACOG, laboratory and clinical guidelines for populationbased CF screening were published in 2001. Fortunately, the ACMG Committee informally distributed its proposed recommended screening panel several months before publication and 5 months before the announcement of these recommendations in this Journal. This lead time was important for laboratories and commercial vendors to develop appropriate tools to begin such a program. As with all such wide reaching programs, continuing reassessment will be necessary to ensure that the program is fulfilling the needs of patients without causing harm. 
Our initial experience screening more than 20,000 patients using the ACMG recommended panel has raised some compelling issues, especially with regard to the I148T mutation and the IVS8-5T/7T/9T polymorphism. We observed a more than 50 -fold higher prevalence of the I148T mutation $(6.8 \%$ of CF chromosomes) in our population of predominantly carrier screens compared with studies of CF patient populations. This discrepancy is much greater than the $\mathrm{R} 117 \mathrm{H}$ mutation known to be a mild mutation with incomplete penetrance. The increased prevalence of the I148T is consistent with preliminary data by Rohlfs et al., ${ }^{5}$ who report I148T to account for $6.4 \%$ of CF chromosomes in a population screened for carrier detection. Rohlfs and colleagues' report also identified five unaffected individuals with genotypes I148T/I148T or delta F508/ I148T. We also discovered two asymptomatic adult patients with the genotype delta F508/I148T. However, five symptomatic patients were identified who are compound heterozygotes for I148T and another CF mutation in the Rohlfs study. Rohlfs performed linkage analysis revealing that there are at least two haplotypes upon which the I148T mutation occurs, but these haplotypes are not predictive of affected status. Bozon has contributed to the $\mathrm{CF}$ electronic database a CF chromosome that contains both I148T and 3199del6, a 6 base pair deletion. ${ }^{3}$ The potential significance of this finding with respect to the expression of I148T should be investigated. The incomplete penetrance of I148T should be incorporated into genetic counseling sessions for patients found to carry this allele.

The Hispanic mutation D1270N was not included in the ACMG recommendations. In a limited series of 1,040 patients, this mutation had a $>100$-fold higher than expected prevalence among CF chromosomes. This observation would suggest that it would be unwise to add the D1270N mutation to the standard panel until its penetrance has been determined.

The ACMG recommendations specify reporting the results for the IVS8-5T/7T/9T polymorphism only as a reflex in the presence of $\mathrm{R} 117 \mathrm{H}$. We endorse this recommendation to avoid encouraging prenatal diagnosis for the IVS8-5T genotype. Because some clinicians prefer to know the IVS8-5T status of their patients under any circumstances, we now offer two separate tests, a prenatal carrier screen and an infertility/linkage test. The ACMG recommends reporting the IVS8-5T only as a reflex in the presence of $\mathrm{R} 117 \mathrm{H}$ primarily to evaluate the potential for CBAVD. It may be more prudent to never report the polymorphism when CF testing is performed for the purposes of carrier screening. We believe that this would prevent the performance of an invasive prenatal diagnostic procedure solely for the purpose of genotyping the IVS8-5T allele.

\section{ACMG versus extended panels}

More than 1,000 distinct CF mutations have been reported. ${ }^{3}$ Debates continue regarding how many mutations should be analyzed in a screening program and under what circumstances a more extended panel than the ACMG recommendations should be performed. Each approach has its advantages and disadvantages. The advantage of using a core panel such as the ACMG screening recommendations is that there is now one commercially available kit (and soon there will be others) capable of reliably identifying all the mutations in a timely, cost-effective manner. The current turnaround times in our laboratory are 3 to 4 days after receipt of sample to final report. In pregnancy, timeliness of results is imperative. In addition, the incremental yield for adding extra mutations is minimal (for review see Grody et al. ${ }^{1}$ ). In our platform comparison, the OLA kit assayed an additional nine mutations to the ACMG panel and no chromosomes were detected with those mutations in 1,040 patients. In the Heim study, 93 mutations were analyzed and 29 were not detected at all in 5,840 CF chromosomes and another 7 were detected only once. ${ }^{4}$ Given that the highest CF carrier frequency in any population is approximately 1:25, the expected frequency of any of these rare mutations in a carrier screened population would be around 1 in 150,000 .

Another difficulty with extended panel screening is the paucity of information regarding the penetrance of these minor alleles. From our data it is now clear that I148T is a low penetrance allele and that $\mathrm{D} 1270 \mathrm{~N}$ is probably a low penetrance allele. It is possible that some of these other alleles that are found infrequently in CF patients may have a much higher prevalence in the general population. If only a few laboratories are screening for this allele, it may take years to accumulate sufficient statistics to make such an observation. This delay could lead to patients having invasive prenatal diagnostic procedures and potentially terminating unaffected fetuses. Another benefit of the ACMG panel is that, because all laboratories are screening for the same panel, statistics can be generated quickly and the panel modified or patients counseled regarding penetrance issues.

An additional problem with extended panel testing is the scarcity of genomic DNA samples of known heterozygotes available to develop and validate assays for these rare mutations. Many laboratories have been forced to use synthetic templates as controls to validate assays, which is a risky practice. We have observed situations for which synthetic controls behave very differently from genomic DNA samples. While optimizing a chip-based assay for the ACMG panel, we used a synthetic oligonucleotide for a mutation for which we had no genomic control. The assay was performing well for this mutation when we detected a carrier in our testing program. When genomic DNA from this individual was tested on our chip, the assay did not detect the mutation, despite the fact that the synthetic control gave an excellent signal. This phenomenon may be due to stoichiometry or polymorphism problems and emphasizes the importance of using true genomic controls for assay development. Obtaining genomic controls for these rare mutations would be extremely difficult.

Finally, many strategies for extended panel screening have longer turnaround times, especially when a mutation is detected. These prolonged turnaround times can be problematic during pregnancy, especially if sequential screening has been used. Extended panels are clearly indicated for CF patients and their relatives who have one or both mutations unidentified by standard screening. 


\section{Strom et al.}

The ACMG recommended panel is appropriate for screening pregnant populations and as an initial screen for Caucasian CF patients. For Ashkenazi Jewish CF patients, 94\% will have both mutant alleles identified and for Northern European patients that number is $78 \% .{ }^{11}$ For CF patients of other races, or those with undetected mutations, and for relatives of such patients, extended screening may be beneficial. Newer gene scanning techniques using denaturing high performance liquid chromatography $^{12}$ or complete CFTR gene exon and splice junction sequencing should theoretically be able to identify more than $95 \%$ of the more than 1000 reported CF mutations. Even these tests cannot guarantee that a rare mutation will not be missed. These tests would not be appropriate for carrier screening, because previously unreported sequence variations will be discovered whose significance may be difficult or impossible to predict.

In summary, our experience has demonstrated that it is possible to perform accurate, high throughput CF screening for the ACMG panel with a rapid turnaround time using commercially available ASR reagents. Our data also demonstrate that the I148T allele is a low-penetrance CF allele and that D1270N is probably another such allele.

\section{References}

1. Grody WW, Cutting GR, Klinger KW, Richards CS, Watson M, Desnick RJ. Laboratory standards and guidelines for population-based cystic fibrosis carrier screening. Genet Med 2001;3:149-154.

2. American College of Obstetrics and Gynecology, and American College of Medical Genetics. Preconception and prenatal carrier screening for Cystic Fibrosis. Clinical and laboratory guidelines. Washington, DC: American College of Obstetrics and Gynecology, 2001.

3. Hospital for Sick Children, Toronto, Cystic Fibrosis Mutation Database, http:// www.genet.sickkids.on.ca/cftr/

4. Heim RA, Sugarman EA, Allitto BA. Improved detection of cystic fibrosis mutations in the heterogeneous U.S. population using an expanded pan-ethnic panel. Genet Med 2001;3:168-176.

5. Rohlfs EM, Zhou Z, Sugarman EA, Heim EI, Pace RG, Knowles MR, Silverman LM, Allitto BA. The I148T CFTR mutation confers a variable phenotype and occurs on multiple haplotypes. Paper presented at the 2001 Annual Meeting of the American Association of Human Genetics [abstract 2600]. Abstract appears in Am J Hum Genet Suppl 2001;69.

6. Rave-Harel N, Kerem E, Nissim-Rafinia M, Madjar I, Goshen R, Augarten A, Rahat A, Hurwitz A, Darvasi A, Kerem B. The molecular basis of partial penetrance of splicing mutations in cystic fibrosis. Am J Hum Genet 1997;60:87-94.

7. Chu CS, Trapnell BC, Curristan S, Cutting GR, Crystal RG. Genetic basis of variable exon 9 skipping in cystic fibrosis transmembrane regulator mRNA. Nat Genet 1993; 3:151-156.

8. Niksik M, Romano M, Buratti E, Pagnini F, Baralle FE. Functional analysis of cisacting elements regulating the alternative splicing of human CFTR exon 9. Hum Mol Genet 1999;8:2339-2349.

9. Chillon M, Casals T, Bassas L, Lissens W, Silber S, Romey MC, Ruiz-Romero J, Verlingue C, Claustres M, Nunes V, Ferec C, Estivill X. Mutations in the cystic fibrosis gene in patients with congenital absence of the vas deferens. $N$ Engl J Med 1995;332:1474-1480

10. Costes B, Giroden E, Ghanem N, Fiori E, Jardin A, Souffir JC, Goosens M. Frequent occurrence of the CFTR intron 8 (TG)n 5T allele in men with congenital absence of the vas deferens. Eur J Hum Genet 1995;3:285-293.

11. Palomaki GE, Haddow JE, Bradley LA, FitzSimmons SC. Updated assessment of cystic fibrosis mutation frequencies in non-Hispanic Caucasians. Genet Med 2002; 4:90-94.

12. Le Marechal C, Audrezet MP, Quere I, Raguenes O, Langonne S, Ferec C. Complete and rapid scanning of the cystic fibrosis transmembrane regulator (CFTR) by denaturing high-performance liquid chromatography (D-HPLC): major implications for genetic counseling. Hum Genet 2001:108:290-298. 